\title{
Factores de riesgo cardiovascular en pacientes hospitalizados por COVID-19
}

\author{
Cardiovascular risk factors in patients hospitalized for COVID-19 \\ Fatores de risco cardiovascular em pacientes hospitalizados por COVID-19
}

Henry Mejía-Zambrano,2a

https://orcid.org/0000-0001-7325-779658X

\section{Resumen}

Objetivo: Determinar los factores de riesgo cardiovascular en pacientes hospitalizados por COVID-19. Material y métodos: Revisión sistemática que incluyó estudios observacionales con factores de riesgo de la enfermedad cardiovascular en pacientes hospitalizados por COVID-19, con texto disponible, en idioma inglés o español; se excluyeron cartas al editor, resúmenes, documentos y ensayos clínicos, estudios sin datos confiables y fuentes duplicadas. Se realizó una búsqueda bibliográfica de literatura en PubMed, MEDLINEy JAMA, del 12 al 18 de junio de 2021, se evaluó estrategias de búsqueda mediante el programa del Manual Cochrane de revisiones sistemáticas. Se utilizó el programa Mendeley para gestionar las referencias bibliográficas y eliminación de estudios. Se evaluó la calidad metodológica de artículos con herramientas del Instituto Nacional del Corazón, Pulmones y Sangre (NHLBI), y se proporcionó una calificación general $\geq 75 \%$ bueno, $50-75 \%$ regular, < 50 \% malo. Resultados: De 400 artículos se seleccionaron 10 estudios de cohortes, 3 retrospectivos, 1 prospectivo y 1 revisión sistemática y metaanálisis. Los factores de riesgo de la enfermedad cardiovascular en pacientes hospitalizados con COVID-19, en esta revisión sistemática fueron: el $46 \%$ de los pacientes padeció hipertensión arterial (HTA); el $21 \%$, diabetes mellitus; el $15 \%$, dislipidemia; el 10 $\%$, enfermedad renal crónica (ERC); el $6 \%$, infarto miocardio (IAM); el $5 \%$, obesidad mórbida; el $4 \%$, insuficiencia cardiaca crónica (ICC) y fibrilación auricular (FA); y el $3 \%$, enfermedad cerebrovascular (ECV). Conclusión: Los principales factores de riesgo de la enfermedad cardiovascular en pacientes hospitalizados por COVID-19 fueron: hipertensión arterial, diabetes mellitus, dislipidemia, infarto de miocardio y obesidad.

Palabras clave: COVID-19, factor de riesgo, enfermedad cardiovascular (Fuente: DeCS)

\section{Abstract}

Objective: To determine cardiovascular risk factors in patients hospitalized for COVID-19. Material and methods: Systematic review that included observational studies with cardiovascular disease risk factors in patients hospitalized with COVID-19, with text available, in English or Spanish; letters to the editor, abstracts, documents and clinical trials were excluded, studies without reliable data and duplicate sources. A bibliographic search of literature was conducted in PubMed, MEDLINE and JAMA, from June 12 to 18, 2021, search strategies were evaluated through the program of the Cochrane Manual of systematic reviews. The Mendeley program was used to manage bibliographic references and eliminate studies. The methodological quality of articles was evaluated with tools from the National Heart, Lung and Blood Institute (NHLBI), and an overall rating of $75 \%$ good, $50-75 \%$ regular, $<50 \%$ bad was provided. Results: From 400 articles, 10 cohort studies, 3 retrospective studies, 1 
prospective study, and 1 systematic review and meta-analysis were selected. The risk factors for cardiovascular disease in patients hospitalized with COVID-19 in this systematic review were: $46 \%$ of patients had high blood pressure (HTN); $21 \%$ had diabetes mellitus; $15 \%$ had dyslipidemia; $10 \%$ had chronic kidney disease (CKD); 6\%, myocardial infarction (AMI); 5\%, morbid obesity; 4\%, chronic heart failure (CHF) and atrial fibrillation (AF); and $3 \%$, cerebrovascular disease (CVD). Conclusion: The main risk factors for cardiovascular disease in patients hospitalized for COVID-19 were: hypertension, diabetes mellitus, dyslipidemia, myocardial infarction and obesity.

Keywords: COVID-19, risk factors, cardiovascular disease (Source: MeSH-NCBI)

\section{Resumo}

Objetivo: Determinar os fatores de risco cardiovascular em pacientes hospitalizados pela COVID-19. Material e métodos: Revisão sistemática que incluiu estudos observacionais com fatores de risco da doença cardiovascular em pacientes hospitalizados por COVID-19, com texto disponível, em inglês ou espanhol; foram excluídas cartas ao editor, resumos, documentos e ensaios clínicos, estudos sem dados confiáveis e fontes duplicadas. Foi realizada uma busca bibliográfica de literatura na PubMed, MEDLINE e JAMA, de 12 a 18 de junho de 2021, e foram avaliadas estratégias de busca através do programa do Manual Cochrane de revisões sistemáticas. O programa Mendeley foi utilizado para gerenciar as referências bibliográficas e a eliminação de estudos. Avaliou-se a qualidade metodológica de artigos com ferramentas do Instituto Nacional do Coração, Pulmões e Sangue (NHLBI), e se proporcionou uma qualificação geral $75 \%$ bom, 50-75 \% regular, < $50 \%$ mau.

Resultados: De 400 artigos foram selecionados 10 estudos de coortes, 3 retrospectivos, 1 prospectivo e 1 revisão sistemática e metaanálise. Os factores de risco da doença cardiovascular em pacientes hospitalizados com COVID-19, nesta revisão sistemática foram: $46 \%$ dos pacientes padeceu de hipertensão arterial (HTA); $21 \%$, diabetes mellitus; $15 \%$, dislipidemia;
$10 \%$, doença renal crónica (ERC); $6 \%$, infarto miocárdio (IAM); $5 \%$, obesidade mórbida; $4 \%$, insuficiência cardíaca crônica (ICC) e fibrilação auricular (FA); e $3 \%$, doença cerebrovascular (ECV). Conclusão: Os principais fatores de risco da doença cardiovascular em pacientes hospitalizados por COVID-19 foram hipertensão arterial, diabetes mellitus, dislipidemia, infarto do miocárdio e obesidade.

Palavras-chave: COVID-19, fator de risco, doença cardiovascular (Fonte: DeCS)

\section{Introducción}

En las últimas dos décadas se evidenció brotes de coronavirus, como el SARS-CoV-1 y el MERS-CoV en los años 2002 y 2012, respectivamente. A finales del año 2019, surgió el brote de un nuevo coronavirus desencadenando el síndrome respiratorio severo agudo coronavirus 2 (SARSCoV-2), que ocasiona la enfermedad del coronavirus 2019 (COVID-19), originado en la provincia de Hubei, de la ciudad de Wuhan, en la República Popular de China, causando una pandemia a nivel mundial. ${ }^{1,2}$ La Organización Mundial de la Salud (OMS) declaró pandemia el 11 de marzo del 2020, ocasionando un gran problema en la salud pública a nivel mundial. Hasta el 13 de junio de 2021, la OMS reportó 175 306598 casos confirmados y 3792777 decesos. ${ }^{3}$

En un estudio, se observó que las personas con enfermedades subyacentes cardiovascular estuvieron predispuestos a la infección respiratoria pandémica del MERS-CoV (2012), donde el $30 \%$ corresponde a enfermedades cardiacas y el $16 \%$ a la obesidad, que provocaron una elevada tasa de morbilidad o mortalidad en todo el mundo, debido a la elevada síntesis de citoquinas proinflamatorias y deterioran los sistemas inmunológicos innatos y adquiridos del huésped. 4.5 Algunos estudios mostraron un análisis en pacientes con COVID-19, donde sugieren que los pacientes con alguna enfermedad cardiovascular subyacente, tuvieron un mayor riesgo de desarrollar síntomas graves y de mal pronóstico, siendo las tasas altas de mortalidad en los pacientes con hipertensión arterial (HTA) y enfermedad cerebrovascular 
(ECV). ${ }^{6}$ Las tasas de hospitalización en pacientes infectados por COVID-19 fueron altas en los pacientes de 65 años o más y entre los pacientes adultos jóvenes, la mayoría tenían una o dos afecciones subyacentes, HTA y ECV. Además, existen otras comorbilidades o enfermedades subyacentes cardiovascular en los pacientes con COVID-19, como la HTA y diabetes mellitus, que los llevaron a hospitalización y asistencia ventilatoria. ${ }^{8}$ La alta incidencia de ECV en los pacientes infectados por COVID-19, puede predisponer a mayores complicaciones, ya que los pacientes con ECV subyacente son vulnerables y puede provocar la muerte. ${ }^{9}$ En esta revisión sistemática, se determinará los factores de riesgo cardiovascular en pacientes hospitalizados por COVID-19.

\section{Material y métodos}

Este informe de revisión sistemática, se realizó conforme a los ítems de referencia para publicar protocolos de revisión sistemática y metaanálisis (PRISMA) (http://www.prisma-statement.org/).

\section{Criterios de elegibilidad}

En esta revisión sistemática se incluyeron a) estudios observacionales, b) estudios con factores de riesgo de la enfermedad cardiovascular en pacientes hospitalizados por COVID-19, c) estudios con texto disponible y d) estudios en idioma inglés o español. Se excluyeron a) cartas al editor, resúmenes, documentos y ensayos clínicos, b) estudios con factores de riesgo de la enfermedad cardiovascular en pacientes por COVID-19 no hospitalizados, c) estudios sin datos confiables, d) fuentes duplicadas, y e) estudios distintos a los idiomas del español e inglés.

\section{Búsqueda o estrategia de búsqueda}

Se realizó una búsqueda bibliográfica de literatura en PubMed, MEDLINE y JAMA, del 12 de junio hasta el 18 de junio de 2021. El autor elaboró las estrategias de búsqueda, según las recomendaciones del Manual Cochrane de revisiones sistemáticas. Se empleó términos de búsqueda de vocabulario controlado para MEDLINE (MeSH), enlazados en término de texto para cada uno de los conceptos seleccionados mediante operadores booleanos: "COVID-19", "SARS-COV-2", "CARDIOVASCULAR DISEASE AND COVID-19","RISK FACTORS AND CARDIOVASCULAR AND COVID-19". No se emplearon filtros de fecha ni restricción de formato del documento de búsqueda. Se realizaron estrategias de búsqueda, con la herramienta de búsqueda avanzada en la base de datos, antes de aprobar un consenso final de las estrategias de búsqueda. La estrategia de búsqueda completa está en el material suplementario de este informe de revisión. Se utilizó el programa Mendeley para gestionar referencias bibliográficas y para la eliminación de artículos duplicados.

\section{Selección de estudios y extracción de datos}

La revisión fue realizada por el autor. Se recuperaron los textos completos de los artículos para determinar la elegibilidad y verificar la lista de inclusión y exclusión. Se utilizó el programa Microsoft Excel para la extracción, almacenamiento y el análisis de datos. Se extrajeron los siguientes datos: autor, referencia, país, población de estudio, calidad del estudio, diseño de estudio, resultados de los factores de riesgo.

\section{Síntesis de resultados}

Se realizó una síntesis narrativa formal de los datos recogidos, no se realizó una síntesis estadístico formal. La síntesis se centró en el análisis cualitativo de las manifestaciones clínicas mencionadas, en cada uno de los países de los estudios publicados.

\section{Evaluación de la calidad de estudio}

Se evaluó la calidad metodológica de los artículos utilizando las herramientas de evaluación de la calidad del Instituto Nacional del Corazón, los Pulmones y la Sangre (NHLBI). ${ }^{10}$ Cada herramienta contiene criterios que evalúan la validez interna y el riesgo de sesgo. Los criterios se evaluaron como "Sí", "No" u "Otro" y se proporcionó una calificación general para cada estudio de los elementos calificados con una respuesta afirmativa: $\geq 75 \%=$ bueno, $50-75 \%=$ regular,$<50 \%=$ malo. 


\section{Resultados}

\section{Selección y características de los estudios}

En la búsqueda inicial se identificaron 400 resultados. Se eliminaron 250 resultados duplicados. Después de filtrar por títulos y resúmenes evaluamos 85 artículos en texto completo. Se registraron inicialmente 13 artículos que brindan información específica relacionada con los objetivos de este estudio.
Además, se realizó una búsqueda secundaria de los 13 estudios incluidos inicialmente, de lo cual se incorporaron 2 estudios adicionales, resultando 15 estudios. Finalmente, se adjuntaron 15 estudios: 10 estudios de cohorte, 3 estudios retrospectivos, 1 estudio prospectivo y un estudio de revisión sistemática y metaanálisis, para su posterior análisis y 10 estudios sobre comentarios para esta revisión (Figura 1).

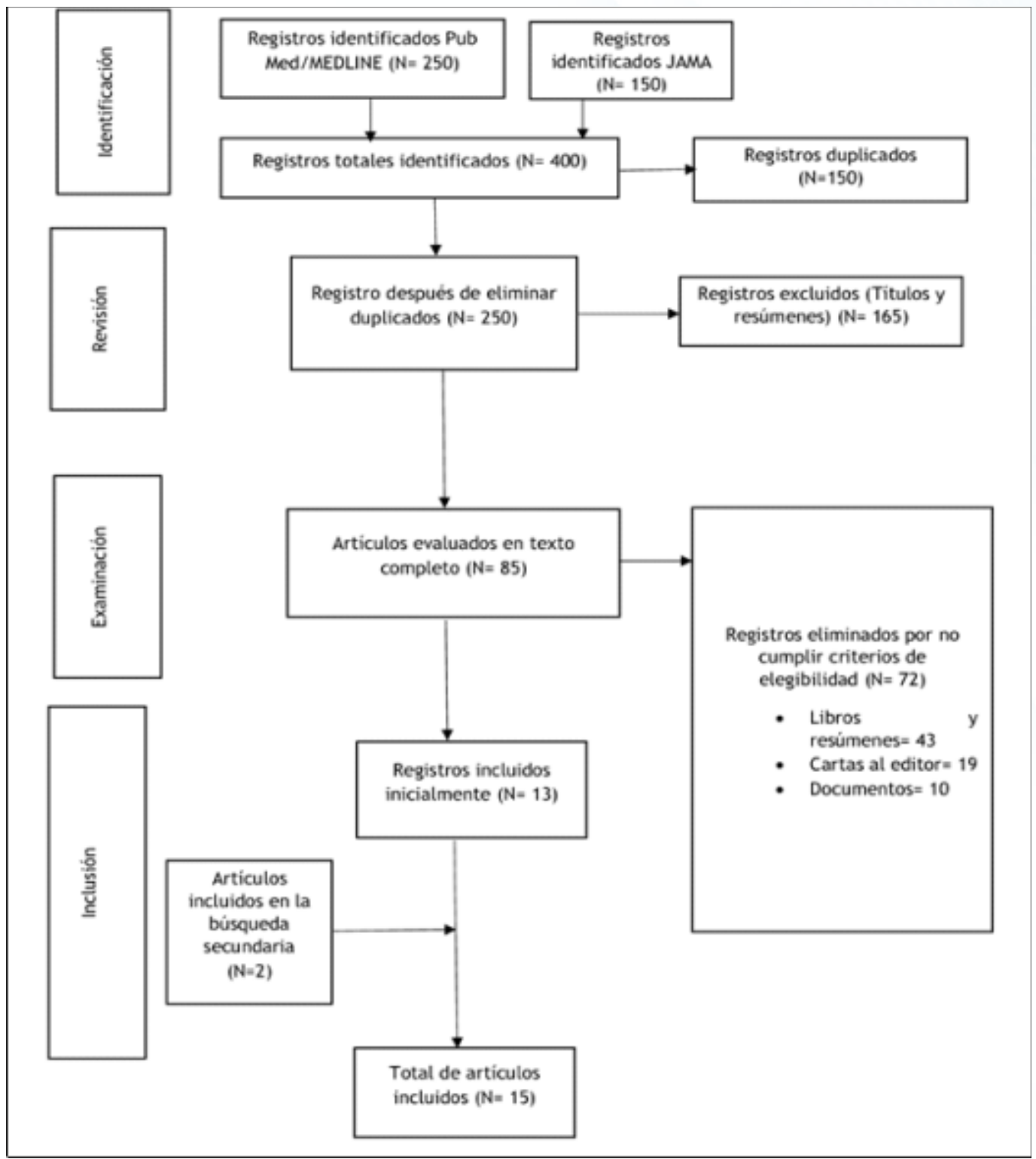

Figura 1. Flujograma de selección de artículos

La mayoría de los artículos provienen de China (5/15), Italia (2/15), USA (4/10), España (1/15), Francia (1/15), Suiza (1/15) y Arabia Saudita (1/15). El total de la población de este estudio fue de 5604 pacientes. La mayoría fueron hombresy la edad promedio fue de 60 años, de los diferentes estudios (Tabla 1). 
Tabla 1

Resultados de los factores de riesgo cardiovascular y COVID-19

\begin{tabular}{|c|c|c|c|c|c|c|}
\hline $\begin{array}{l}\text { Autor } \\
\text { (\# ref) }\end{array}$ & País & $\begin{array}{l}\text { Población } \\
\text { (N) }\end{array}$ & $\begin{array}{l}\text { Características } \\
\text { de la población }\end{array}$ & $\begin{array}{l}\text { Diseño del } \\
\text { estudio }\end{array}$ & $\begin{array}{l}\text { Calidad } \\
\text { del } \\
\text { estudio }\end{array}$ & $\begin{array}{c}\text { Resultados de } \\
\text { los factores de } \\
\text { riesgo cardiovascular (\%) }\end{array}$ \\
\hline $\begin{array}{l}\text { Xu L. et al. } \\
\text { (11) }\end{array}$ & China & 2032 & $\begin{array}{c}M=1524 \\
F=508 \\
\text { Edad } \begin{array}{c}\text { media }=54,4 \\
\text { años }\end{array}\end{array}$ & Metaanálisis & regular & $\begin{array}{l}\operatorname{HTA}(47,1 \%) \\
\operatorname{ECV}(25,7 \%) \\
\operatorname{DM}(20,4 \%)\end{array}$ \\
\hline $\begin{array}{l}\text { Puntmann } \\
\text { V. } \\
\text { et al. (12) }\end{array}$ & Italia & 100 & $\begin{array}{c}M=53 \\
F=47 \\
\text { Edad media }=49 \pm \\
14 \text { años }\end{array}$ & $\begin{array}{c}\text { Cohorte } \\
\text { observacional }\end{array}$ & regular & $\begin{array}{c}\text { HTA }(22,2 \%) \\
\text { Dislipidemia (22 \%) } \\
\text { DM (18\%) } \\
\text { IAM (13\%) } \\
\text { EPOC (21\%) }\end{array}$ \\
\hline $\begin{array}{l}\text { Guo T. et al. } \\
\text { (13) }\end{array}$ & China & 187 & $\begin{array}{c}M=91 \\
F=96 \\
\text { Edad media }= \\
58,50 \text { años }\end{array}$ & Retrospectivo & regular & $\begin{array}{c}\operatorname{HTA}(32,6 \%) \\
\operatorname{DM}(15 \%) \\
\text { IAM }(11,2 \%) \\
\text { IECA/BRA }(10,1 \%) \\
\text { Miocardiopatia }(4,3 \%) \\
\text { ERC }(3,2 \%)\end{array}$ \\
\hline $\begin{array}{l}\text { Rubin G. } \\
\text { et al. (14) }\end{array}$ & USA & 965 & $\begin{array}{c}M=561 \\
F=404 \\
\text { Edad } \begin{array}{c}\text { media }=65 \\
\text { años }\end{array}\end{array}$ & $\begin{array}{c}\text { Cohorte } \\
\text { observacional }\end{array}$ & regular & $\begin{array}{c}\text { HTA }(48,6 \%) \\
\text { DM }(32,1 \%) \\
\text { Obesidad mórbida (18,8\%) }\end{array}$ \\
\hline $\begin{array}{l}\text { Purroy F. } \\
\text { et al. (15 }\end{array}$ & España & 1737 & $\begin{array}{c}M=686 \\
F=1051 \\
\text { Edad media }=65,5 \\
\text { años }\end{array}$ & Transversal & regular & $\begin{array}{c}\text { HTA }(37,2 \%) \\
\text { Dislipidemia }(22,5 \%) \\
\text { DM }(15,1 \%) \\
\text { ERC }(3,7 \%) \\
\text { ICC }(2,2 \%) \\
\text { FA }(4,1 \%) \\
\text { IAM }(5,2 \%) \\
\text { ECV }(0,9 \%) \\
\end{array}$ \\
\hline $\begin{array}{l}\text { Lodigiani C. } \\
\text { et al. (16) }\end{array}$ & Italia & 388 & $\begin{array}{c}\qquad \begin{array}{c}M=312 \\
F=76 \\
\text { Edad } \text { media }=66 \\
\text { años }\end{array}\end{array}$ & $\begin{array}{c}\text { Cohorte } \\
\text { observacional }\end{array}$ & regular & $\begin{array}{c}\text { HTA (47,2\%) } \\
\text { DM (22,7\%) } \\
\text { Obesidad mórbida (24,1\%) } \\
\text { Dislipidemia (19,6\%) } \\
\text { ERC }(15,7 \%) \\
\end{array}$ \\
\hline $\begin{array}{l}\text { Panagides } \\
\text { V. et al. (17) }\end{array}$ & Francia & 2809 & $\begin{array}{c}M=1618 \\
F=1191 \\
\text { Edad media }=66,4 \\
\pm 16,9 \text { años }\end{array}$ & $\begin{array}{c}\text { Cohorte } \\
\text { observacional }\end{array}$ & regular & $\begin{array}{c}\text { HTA }(50,6 \%) \\
\text { Obesidad mórbida (30,3\%) } \\
\text { Dislipidemia (27,9\%) } \\
\text { DM }(23,7 \%) \\
\text { FA }(14,3 \%) \\
\text { ERC }(13,9 \%) \\
\text { IAM }(12,4 \%) \\
\text { EV }(3,98 \%) \\
\operatorname{ICC}(5,73 \%) \\
\operatorname{ETV}(7,48 \%)\end{array}$ \\
\hline $\begin{array}{l}\text { Rosenthal N. } \\
\text { et al. (18) }\end{array}$ & USA & 64781 & $\begin{array}{c}\quad M=31937 \\
F=32844 \\
\text { Edad media }=57 \\
\text { años }\end{array}$ & $\begin{array}{c}\text { Cohorte } \\
\text { observacional }\end{array}$ & regular & $\begin{array}{c}\text { HTA }(46,7 \%) \\
\text { Dislipidemia }(28,9 \%) \\
\operatorname{DM}(16,8 \%) \\
\operatorname{ERC}(14 \%) \\
\text { ICC }(9,3 \%) \\
\operatorname{IAM}(5,7 \%) \\
\operatorname{ECV~}(4,6 \%) \\
\operatorname{EAP}(2,6 \%)\end{array}$ \\
\hline $\begin{array}{l}\text { Ryffel C. et } \\
\text { al. (19) }\end{array}$ & Suiza & 71 & $\begin{array}{c}M=39 \\
F=32 \\
\text { Edad } \text { media }=75,9 \\
\text { años }\end{array}$ & $\begin{array}{c}\text { Cohorte } \\
\text { observacional }\end{array}$ & regular & $\begin{array}{c}\text { HTA }(90,5 \%) \\
\text { Dislipidemia }(52,45 \%) \\
\operatorname{DM}(32,2 \%) \\
\text { IAM }(31,2 \%)\end{array}$ \\
\hline
\end{tabular}


$\mathrm{N}$ : número de la población; $\mathrm{M}$ : masculino; F: femenino; HTA: hipertensión arterial; DM: diabetes mellitus; ECV: enfermedad cerebrovascular; IMA: infarto agudo de miocardio; EPOC: enfermedad pulmonar obstructiva crónica; IECA: inhibidor de la enzima convertidora de angiotensina; BRA: bloqueador del receptor de angiotensina; ERC: enfermedad renal crónica; ICC: insuficiencia cardiaca crónica; FA: fibrilación auricular; EV: enfermedad valvular; ETV: enfermedad trombótica venosa; EAP: enfermedad arterial periférica.

Resultados de los factores de riesgo cardiovascular y COVID-19 (Tabla 1)

Xu L. et al. ${ }^{11}$ realizaron un estudio de metaanálisis, donde incluyeron 20 estudios, con una población de 2032 pacientes hospitalizados. Observaron que la edad media fue de 54,4 años, la relación de hombres y mujeres fue de 1:2. Además, las comorbilidades principales fueron: HTA $(47,10 \%)$, ECV $(25,70 \%)$ y diabetes mellitus $(20,40 \%)$.

Puntmann $V$. et al. ${ }^{12}$ realizaron un estudio de cohorte observacional, donde incluyeron a 100 pacientes, 53 hombres y 47 mujeres, la edad media fue de $49 \pm 14$ años. El índice de masa corporal (IMC) promedio fue de $23-28 \mathrm{~kg} / \mathrm{m} 2$. Las principales comorbilidades subyacentes y cardiovasculares fueron: 22 pacientes con HTA (22\%); 18 pacientes con diabetes mellitus (18\%); 22 pacientes con hipercolesterolemia (22\%); 13 pacientes con antecedentes de IAM (13\%); 22 pacientes fumadores crónicos (22\%); y 21 pacientes con una enfermedad pulmonar obstructiva crónica (EPOC) (21\%).

Un estudio observacional retrospectivo realizado en China por Guo T. et al. ${ }^{13}$ incluye 187 pacientes, 91 hombres y 96 mujeres; la edad media fue de 58,50 años. Observaron las siguientes comorbilidades: el 32,6\% de pacientes presentaron HTA; el 11,2\% de pacientes, IAM; el $15 \%$, diabetes mellitus; el 10,1\%, historial de uso de inhibidores de la enzima convertidora de angiotensina (IECA) y bloqueador del receptor de angiotensina (ARB); el $7 \%$, neoplasia maligna; el 4,3\% sufrieron miocardiopatía; y el 3,2 \% tuvieron ERC.
En Estados Unidos (USA), Rubin G. et al. ${ }^{14}$ realizaron un estudio de cohorte, donde incluyeron a 965 pacientes; el 58,1 \% fueron hombres y el $41,9 \%$, mujeres. La edad media fue de 65 años. Observaron en los pacientes lo siguiente: el 48,6 \% padecieron HTA; el 40,7 \% tomaron fármacos con elevación prolongada del intervalo QTc; el 32,1\% padecieron diabetes mellitus;y el 18,8\% tuvieron obesidad mórbida.

En España, Purroy F. et al. ${ }^{15}$ realizaron un estudio transversal, donde incluyeron a 1737 pacientes, el 39,5\% fueron mujeres y el 60,5\%, hombres; la edad media fue de 65,5 años. Observaron que las comorbilidades en los pacientes fueron: el 37,2\% tuvieron HTA; el 15,1\% padecieron diabetes mellitus; el 22,5\% tuvieron hiperlipidemia; el 3,7 $\%$ estuvieron con ERC; el 2,2 \% con ICC; el 4,1\%, FA; el 5,2\%, IAM; y el 0,9\% padecieron ECV.

En Italia se realizó un estudio de cohorte retrospectivo, realizado por Lodigiani $C$. et al. ${ }^{16}$ donde incluyeron 388 pacientes (327 hospitalizados y 61 en UCI); el 80,3\% fueron hombres y el 19,7\%, mujeres; la edad media fue de 66 años. Además, observaron que los factores de riesgo de los pacientes fueron: el 47,2 \% con HTA; el 22,7 \% tuvieron diabetes mellitus; el 24,1 \% padecieron obesidad mórbida; el 19,6\% tuvieron dislipidemia; el 15,7 \% con ERC; y el 11,6 $\%$ fueron fumadores crónicos.

En un estudio observacional realizado en Francia por Panagides V. et al. ${ }^{17}$ donde incluyeron a 2809 pacientes; el 57,6 \% fueron hombres y el $42,4 \%$, mujeres; la edad media fue de 66,4 \pm 16,9 años. Observaron, que los pacientes con comorbilidad cardiovascular fueron: el 50,6 \% con HTA; el 30,3\% con obesidad mórbida; el 23,7 \% con diabetes mellitus; el 27,9\% con dislipidemia; el 13,4\% eran fumadores; el 13,9\% con ERC; el 14,3\% con FA; el $12,4 \%$ padecieron IAM; el 3,98 \% con enfermedad cardiaca valvular; el 5,73\% con ICC; y el 7,48\% padecieron enfermedad tromboembólica venosa (ETV).

En un estudio de cohorte realizado en USA por Rosenthal N. et al. ${ }^{18}$ donde incluyeron a 64781 pacientes; el 49,3\% fueron hombres y el 50,1\%, mujeres; la edad media fue de 57 años. Observaron, que los pacientes con comorbilidad 
cardiovascular o subyacente fueron las siguientes: el 46,7 \% tuvieron HTA, el 28,9\% padecieron hiperlipidemia, el 16,8\% pacientes con diabetes mellitus no complicada; y el 11,2\% con diabetes complicada crónica; el 16,1 \% con EPOC; el $14 \%$ padecieron ERC; el 9,3\% tuvieron ICC; el 5,7\% con IAM previa, el 4,6\% mostraron una ECV; y el 2,6 \% padecieron una enfermedad arterial periférica (EAP).

En un estudio de cohorte, realizado en Suiza por Ryffel C. et al. ${ }^{19}$ donde incluyeron a 71 pacientes con estenosis aórtica grave sintomática, 39 hombres (54,9\%) y 32 mujeres (45,1\%); la edad media fue de 75,9 años. Las comorbilidades cardiovasculares y subyacentes en los pacientes fueron los siguientes: el 90,5\% padecieron de HTA; el 52,45 \% tuvieron hipercolesterolemia; el $33,2 \%$ tuvieron diabetes mellitus; el 31,2\% pacientes mostraron IAM; el 40,65\% tuvieron ERC;yel 27,8\% tuvieron algún historial de FA.

Un estudio transversal retrospectivo en Arabia Saudita por Alshaikh M. et al. ${ }^{20}$ donde se incluyeron 206 pacientes con riesgo de una enfermedad cardiovascular; 138 hombres (67\%) y 68 mujeres ( $37 \%)$; la edad media fue de 55,3 \pm 15,1 años. Además, observaron que los pacientes presentaron las siguientes comorbilidades cardiovasculares y enfermedades subyacentes: 99 padecieron HTA (48,1\%); 93 tuvieron diabetes mellitus (45,1\%); 17 pacientes con EPOC (8,3\%); 23 pacientes tuvieron IAM (11,2\%); 18 pacientes tuvieron ERC (8,7\%); 19 pacientes mostraron ICC (9,2\%); y 15 pacientes mostraron una ECV (7,3\%).

En el estudio de cohorte longitudinal realizado en USA por Loannou G. et al. ${ }^{21}$ donde incluyeron a 10131 pacientes, 9221 hombres (91\%) y 910 mujeres (9\%), la edad media fue de 63,6 años. Observaron que las comorbilidades cardiovasculares y subyacentes fueron: 6294 pacientes con HTA $(62,1 \%) ; 5630$ pacientes mostraron hiperlipidemia (55,6\%); 3861 pacientes mostraron diabetes mellitus (38,1\%); 2203 pacientes mostraron IAM (21,7\%); 1968 pacientes padecieron obesidad mórbida $(19,4$ \%); 1867 pacientes padecieron una ERC (18,4\%);
1125 pacientes mostraron ICC (11,1\%); y 361 pacientes tuvieron una ECV $(3,6 \%)$.

El estudio de cohorte que realizaron en China por Shi S. et al. ${ }^{22}$ donde incluyeron 229 pacientes hospitalizados por lesión cardiaca, 99 hombres (43,2\%) y 130 mujeres (56,8 \%); la edad media fue de 45 años. Los autores observaron que las características de las comorbilidades cardiovasculares fueron: 55 pacientes tuvieron HTA (24\%); 17 pacientes tuvieron diabetes mellitus (7,4\%); 4 pacientes tuvieron IAM (1,7\%); y 2 pacientes mostraron ERC $(0,9 \%)$.

En USA, se realizó un estudio de cohorte por Mortus J. et al. ${ }^{23}$ donde incluyeron a 21 pacientes con síndrome de hipercoagulabilidad y tromboelastrograficos, 12 hombres (57\%) y 9 mujeres (43\%); la edad media fue de 68 años. Observaron que, entre los pacientes, 20 tuvieron una comorbilidad (95\%), el $75 \%$ de los pacientes tuvieron HTA y el $19 \%$ de los pacientes mostraron FAyERC.

En el estudio retrospectivo que analizaron, Sun W. et al. ${ }^{24}$ en pacientes confirmados por COVID-19 en China, donde incluyeron 196 pacientes con lesión cardiaca, 112 hombres (57,1 \%) y 84 mujeres (42,9\%); la edad media fue de 65 años. Observaron que los pacientes con comorbilidad cardiovascular fueron: 87 pacientes padecieron HTA (44,4 \%); 29 pacientes tuvieron diabetes mellitus (14,8\%); 32 pacientes mostraron ICC (16,3\%); 15 pacientes tuvieron una ECV $(7,7 \%) ; 6$ pacientes padecieron ERC $(3,1 \%)$; y 8 pacientes mostraron EPOC $(4,1 \%)$.

En el estudio de cohorte retrospectivo que analizaron, Wu C. et al. ${ }^{25}$ en pacientes confirmados por la proteína C reactiva en tiempo real (PCR-rt) positivo a COVID-19 en China, donde incluyeron a 201 pacientes con neumonía COVID-19, 128 hombres (63,7\%) y 83 mujeres (36,3\%), la edad media fue de 51 años. Las principales comorbilidades cardiovasculares fueron: 39 pacientes tuvieron HTA (19,4\%), 22 pacientes mostraron diabetes mellitus (10,9\%), 8 pacientes con ECV (4\%) y 2 pacientes con ERC ( $1 \%)$. 
Discusión

En esta revisión sistemática se observa que los factores cardiovasculares como la edad, el sexo masculino y las comorbilidades cardiovasculares, como la HTA y diabetes mellitus, son prevalentes en los pacientes con COVID-19. En las diferentes pandemias que azotaron la humanidad, los pacientes con comorbilidad cardiovascular o subyacente, fueron muy vulnerables a las infecciones y posteriormente a la inflamación multisistémica, comprometiendo su estado de salud hasta la muerte. ${ }^{20}$

En este estudio se observó que el $46 \%$ de los pacientes hospitalizados, tuvieron HTA, seguido por el $21 \%$ con diabetes mellitus y otras comorbilidades. Puntmann et al. ${ }^{12}$ encontraron en su estudio que el $22 \%$ de los pacientes tuvieron antecedentes de HTA, seguido de dislipidemia, diabetes mellitus e IAM.

Además, se observó que el $21 \%$ de los pacientes de esta revisión, padecieron diabetes mellitus con y sin complicaciones. En una revisión sistemática y metaanálisis, observaron que una asociación de 20,40\% de pacientes diabéticos no controlados y los malos resultados a favor de las hospitalizaciones o en las unidades de cuidados intensivos (UCl). ${ }^{1}$

En este estudio, se encontró que el $15 \%$ de los pacientes tuvieron dislipidemia, preferentemente, la colesterolemia en los pacientes hospitalizados por la infección de la COVID-19. Panagides et al. ${ }^{17}$ observaron que el $27,9 \%$ de los pacientes padecieron dislipidemia, por detrás de la HTA y diabetes mellitus.

En esta revisión se encontró que el $5 \%$ de los pacientes padecen obesidad mórbida, por detrás de la HTA, diabetes mellitus. Estudios recientes, muestran que la obesidad podría agravar el curso de la COVID-19. ${ }^{18,19}$

Una limitación del estudio fue la calidad de los estudios, con sesgos en la muestra, y en algunos de ellos no se informaron la edad, los factores de riesgo y manifestaciones clínicas. Otra limitación fue la heterogeneidad de los estudios, que podría estar relacionado con los diseños de estudios y el tamaño de la muestra.

\section{Conclusión}

Este estudio concluye que los principales factores de riesgo de la enfermedad cardiovascular en pacientes hospitalizados por COVID-19 fueron: hipertensión arterial, diabetes mellitus, dislipidemia y obesidad.

\section{Declaración de conflicto de intereses}

El autor declara no tener conflictos de intereses con instituciones ni otros autores.

\section{Referencias}

1. Dhama K, Khan S, Tiwari R, Sircar Sh, Bhat S, Malik YS, et al. Coronavirus disease 2019-COVID-19. Clin Microbiol Rev [Internet]. 2020 [citado el 14/06/2021]; 33(4): 1-48. Doi: 10.1128/CMR.00028-20

2. Gralinski LE, Menachery VD. Return of the coronavirus: 2019-nCoV. Viruses [Internet]. 2020 [citado el 14/06/2021]; 12(2): 135. Doi: 10.3390/v12020135.

3. Organización Mundial de la Salud. Tablero COVID-19 [Internet]. Organización Mundial de la Salud; [citado el 07/05/2021]. Disponible: https://covid19.who.int/

4. Badawi A, Ryoo S. Prevalence of comorbidities in the Middle East respiratory syndrome coronavirus (MERS-CoV): a systematic review and metaanalysis. International Journal of Infectious Diseases [Internet]. 2016 [citado el 14/06/2021]; 49: $129-133$. D i s pon i ble: https://pubmed.ncbi.nlm.nih.gov/2735262 8/

5. Badawi A, Ryoo S. Prevalence of comorbidities in the Middle East respiratory syndrome coronavirus (MERS-COV): a systematic review and meta-analysis. International Journal of Infectious Diseases [Internet]. 2016 [citado el 14/06/2021]: 129133 D i s p on i b le: https://pubmed.ncbi.nlm.nih.gov/2735262 8/

6. Wu Z, McGoogan J. Characteristics of and Important Lessons from the Coronavirus Disease 2019 (COVID-19) Outbreak in China: Summary of a Report of 72314 Cases from the Chinese Center for Disease Control and Prevention. JAMA - Journal of the American 
Medical Association [Internet]. 2020 [citado el 14/06/2021]; 323(13): 1239-1242. D i s p o n i b l e : https://jamanetwork.com/journals/jama/ful larticle/2762130

7. Garg S, Kim L, Whitaker M, O'Halloran A, Cummings $C$, Holstein $R$, et al. Hospitalization Rates and Characteristics of Patients Hospitalized with LaboratoryConfirmed Coronavirus Disease 2019 COVID-NET, 14 States, March 1-30, 2020. MMWR. Morbidity and Mortality Weekly Report [Internet]. 2020 [citado el 14/06/2021]; 69(15): 458-464. Disponible: https://pubmed.ncbi.nlm.nih.gov/3229825 1/

8. Alsofayan Y, Althunayyan S, Khan A, Hakawi A, Assiri A. Clinical characteristics of COVID19 in Saudi Arabia: A national retrospective study. Journal of Infection and Public Health [Internet]. 2020 [citado el 14/06/2021]; 13(7): $920-925$. D i s pon i ble: https://pubmed.ncbi.nlm.nih.gov/3253494 5/

9. Yang $X, Y u Y, X u J$, Shu $H, X i a ~ J, ~ L i u ~ H$, et al. Clinical course and outcomes of critically ill patients with SARS-CoV-2 pneumonia in Wuhan, China: a single-centered, retrospective, observational study. The Lancet Respiratory Medicine [Internet]. 2020 [citado el 14/06/2021]; 8(5): 475-481. D i s p o $n$ i b l e : https://pubmed.ncbi.nlm.nih.gov/3210563 2/

10. Instituto Nacional del Corazón, los Pulmones y la Sangre. Herramienta de evaluación de la calidad para estudios observacionales de cohortes y transversales [Internet]. Instituto Nacional del Corazón, los Pulmones y la Sangre; [citado el 07 de mayo de 2021]. D i s p o n i b | e e $n$ : https://www.nhlbi.nih.gov/healthtopics/study-quality-assessment-tools.

11. Xu L, Mao Y, Chen G. Risk factors for 2019 novel coronavirus disease (COVID-19) patients progressing to critical illness: A systematic review and meta-analysis. Aging [Internet]. 2020 [citado el 15/06/2021]; 12(12): 12410-12421. Disponible: https://www.ncbi.nlm.nih.gov/pmc/articles /PMC7343456/
12. Puntmann V, Carerj $M$, Wieters I, Fahim $M$, Arendt C, Hoffmann J, et al. Outcomes of Cardiovascular Magnetic Resonance Imaging in Patients Recently Recovered from Coronavirus Disease 2019 (COVID-19). JAMA Cardiology [Internet]. 2020 [citado el 15/06/2021]; 5(11): 1265-1273. Disponible: https://jamanetwork.com/journals/jamacar diology/fullarticle/2768916

13. Guo T, Fan Y, Chen M, Wu X, Zhang L, He T, et al. Cardiovascular Implications of Fatal Outcomes of Patients with Coronavirus Disease 2019 (COVID-19). JAMA Cardiology [Internet]. 2020 [citado el 15/06/2021]; 5(7): $\begin{array}{lllllll}8 & 1 & 1 & - & 8 & 1 & 8\end{array}$ Disponible:https://jamanetwork.com/journ als/jamacardiology/fullarticle/2763845

14. Rubin G, Desai A, Chai Z, Wang A, Chen Q, Wang A, et al. Cardiac Corrected QT Interval Changes among Patients Treated for COVID19 Infection during the Early Phase of the Pandemic. JAMA Network Open [Internet]. 2021 [citado el 15/06/2021]; 4(4): e216842. D i s p o n i b l e : https://jamanetwork.com/journals/jamanet workopen/fullarticle/2779053

15. Purroy F, Arqué G. Influence of thromboembolic events in the prognosis of COVID-19 hospitalized patients. Results from a cross sectional study. PLOS ONE [Internet]. 2021 [citado el 15/06/2021]; 16(6): e0252351. D i s p o n i b l e : https://www.ncbi.nlm.nih.gov/pmc/articles /PMC8189499/

16. Lodigiani C, lapichino G, Carenzo L, Cecconi M, Ferrazzi P, Sebastian T, et al. Venous and arterial thromboembolic complications in COVID-19 patients admitted to an academic hospital in Milan, Italy. Thrombosis Research [Internet]. 2020 [citado el 15/06/2021]; 191: 9- 14 D i s pon i ble: https://www.ncbi.nlm.nih.gov/pmc/articles /PMC7177070/

17. Panagides $V$, Vincent $F$, Weizman $O$, Jonveaux $M$, Trimaille A, Pommier T, et al. History of heart failure in patients with coronavirus disease 2019: Insights from a French registry. Archives of Cardiovascular Diseases [Internet]. 2021 [citado el 15/06/2021]. $D$ i $s$ p o $n$ i b l e : https://linkinghub.elsevier.com/retrieve/pii/ S1875213621000875 
18. Rosenthal N, Cao Z, Gundrum J, Sianis J, Safo S. Risk Factors Associated with In-Hospital Mortality in a US National Sample of Patients with COVID-19. JAMA Network Open [Internet]. 2020 [citado el 15/06/2021]; 3(12): e $\begin{array}{llllllll} & 2 & 0 & 2 & 9 & 0 & 5 & 8\end{array}$. Disponible:https://jamanetwork.com/journ als/jamanetworkopen/fullarticle/2773971

19. Ryffel C, Lanz J, Corpataux N, Reusser N, Stortecky S, Windecker S, et al. Mortality, Stroke, and Hospitalization Associated with Deferred vs Expedited Aortic Valve Replacement in Patients Referred for Symptomatic Severe Aortic Stenosis during the COVID-19 Pandemic. JAMA Network Open [Internet]. 2020 [citado el 15/06/2021]; 3 (9): e 2020402 . Disponible: https://jamanetwork.com/journals/jamanet workopen/fullarticle/2771036

20. Alshaikh $M$, Alotair $H$, Alnajjar $F$, Sharaf $H$, Alhafi B, Alashgar $L$, et al. Cardiovascular risk factors among patients infected with COVID-19 in Saudi Arabia. Vascular Health and Risk Management [Internet]. 2021 [citado el 15/06/2021]; 17: 161-168. $D$ i s p o n i b l e : https://www.ncbi.nlm.nih.gov/pmc/articles /PMC8071203/

21. Ioannou G, Locke E, Green P, Berry K, O'Hare A, Shah J, et al. Risk Factors for Hospitalization, Mechanical Ventilation, or Death among 10131 US Veterans with SARSCoV-2 Infection. JAMA Network Open [Internet]. 2020 [citado el 15/06/2021]; 3(9): 2022310 . D i s p o n i ble: https://jamanetwork.com/journals/jamanet workopen/fullarticle/2770946

22. Shi S, Qin M, Shen B, Cai Y, Liu T, Yang F, et al. Association of Cardiac Injury with Mortality in Hospitalized Patients with COVID-19 in Wuhan, China. JAMA Cardiology [Internet]. 2020 [citado el 15/06/2021]; 5(7): 802-810. D i s p o n i b l e : https://jamanetwork.com/journals/jamacar diology/fullarticle/2763524

23. Mortus J, Manek S, Brubaker L, Loor M, Cruz $M$, Trautner B, et al. Thromboelastographic Results and Hypercoagulability Syndrome in Patients with Coronavirus Disease 2019 Who Are Critically III. JAMA Network Open [Internet]. 2020 [citado el 15/06/2021]; 3(6): e 2011192 . D i s p o n i ble: https://jamanetwork.com/journals/jamanet workopen/fullarticle/2766786

24. SunW, Zhang Y, Wu C, Wang S, Xie Y, Zhang D, et al. Early vs. Late Onset Cardiac Injury and Mortality in Hospitalized COVID-19 Patients in Wuhan. Frontiers in Cardiovascular Medicine [Internet]. 2021 [citado el 15/06/2021]; 8: 645587. Disponible: https://www.frontiersin.org/articles/10.338 9/fcvm.2021.645587/full

25. Wu C, Chen X, Cai Y, Xia J, Zhou X, Xu S, et al. Risk Factors Associated with Acute Respiratory Distress Syndrome and Death in Patients with Coronavirus Disease 2019 Pneumonia in Wuhan, China. JAMA Internal Medicine [Internet]. 2020 [citado el 15/06/2021]; 180(7): 934-943. Disponible: https://jamanetwork.com/journals/jamaint ernalmedicine/fullarticle/2763184

\section{Correspondencia:}

henrymejiazambrano@gmail.com

Fecha de recepción: 24/8/2021

Fecha deaceptación:22/11/2021 